\title{
Neurological Compromise Associated With COVID-19 Is Broader Than Anticipated
}

\section{Comment on "COVID-19 Pandemic and Neurological Disease: A Critical Review of the Existing Literature"}

\author{
Josef Finsterer ${ }^{1 *}$, Fulvio A. Scorza ${ }^{2}$, Carla A. Scorza ${ }^{2}$, Ana C. Fiorini ${ }^{3,4}$ \\ ${ }^{1}$ Klinik Landstrasse, Messerli Institute, Vienna, Austria \\ ${ }^{2}$ Disciplina de Neurociência, Universidade Federal de São Paulo/Escola Paulista de Medicina (UNIFESP/EPM), São \\ Paulo, Brazil \\ ${ }^{3}$ Programa de Estudos Pós-Graduado em Fonoaudiologia, Pontifícia Universidade Católica de São Paulo (PUC-SP), \\ Brazil \\ ${ }^{4}$ Departamento de Fonoaudiologia, Escola Paulista de Medicina/Universidade Federal de São Paulo (EPM/UNIFESP), \\ São Paulo, Brazil
}

*Corresponding Author: Josef Finsterer, M.D., Ph.D., Klinik Landstrasse, Messerli Institute, Postfach 20, 1180 Vienna, Austria. Tel: +43-1-71165-72085, Fax: +43-1-71165, Email: fifigs1@yahoo.de

I nterested, we read the review article by Assari et al on neurological involvement in coronavirus disease 2019 (COVID-19). ${ }^{1}$ The authors concluded that the review "can help research, public health, and clinical care of COVID-19 patients and reduce morbidity and mortality as well as the economic pressure of the COVID-19 on patients, health care systems, and the society". ${ }^{1}$ We have the following comments and concerns.

The main shortcoming of the study is that several neurological conditions associated with the SARS-CoV-2 infection have not been addressed. Central nervous system abnormalities not included were sinus venous thrombosis, intracerebral bleeding, subarachnoid bleeding, vasoconstriction syndrome, pituitary apoplexia, cerebral vasculitis, encephalitis, acute, haemorrhagic, necrotic encephalopathy, acute demyelinating encephalomyelitis, epilepsy (seizures), and myelitis. ${ }^{2,3}$ Among the peripheral nervous system abnormalities associated with SARS-CoV-2, the authors did not discuss dysgeusia, polyradiculitis, myasthenic syndrome, myositis and rhabdomyolysis.

A further shortcoming is that neurological disorders due to side effects of the drugs given to treat COVID-19 or complications of the treatment were not addressed. Neurotoxic remedies may induce critical ill neuropathy, critical ill myopathy, polyneuropathy or rhabdomyolysis. ${ }^{4}$ Neurotoxic drugs frequently applied in the treatment of COVID-19 patients include chloroquine, steroids and azithromycin. ${ }^{4}$ Long-term pulmonary compromise with or without mechanical ventilation may cause cerebral hypoxia. Nosocomial infections during ICU treatment may trigger sepsis with septic encephalopathy. It is crucial to consider the side effect of drugs applied or complications of treatment as causative for neurological compromise to delineate these secondary abnormalities from direct or indirect primary neurological manifestations of the viral infection with regard to treatment and outcome.

Concerning the therapeutic management of neurological disease in COVID-19 patients, there is only limited discussion on how to manage immunologic neurological disease treated with immunosuppressants, how to manage ischemic stroke due to hypercoagulability, and how to treat newly occurring, so far untreated, immunologic neurological disease.

Though the authors contend that only few original data are available about neurological disease associated with COVID-19, they provide still another review rather than contributing to original data. It is to some extent comprehensible why original data about neuro-COVID-19 are still scarce, given the fact that the pulmonary aspects dominate the diagnostic and therapeutic management. Thus, we would like to appreciate the efforts of those who strived to collect and evaluate data about neurological involvement in cohorts or even single patients with COVID-19. Given the difficult conditions under which COVID-19 patients are managed in hospitals, ambulatory units, nursing homes, or hospices in various areas

Copyright $($ C 2020 The Author(s). This is an open-access article distributed under the terms of the Creative Commons Attribution License (http:// creativecommons.org/licenses/by/4.0), which permits unrestricted use, distribution, and reproduction in any medium, provided the original work is properly cited. 
worldwide, we can of course demand more original data. Yet, we should be happy with the growing amount of evidence that the neurologist has already possessed to play a role in the management of this global burden, which is frequently misused by policymakers to meet their interests and not those of the infected and of those who suffer from the restrictions.

Overall, the review by Assari et al has a number of shortcomings, which should be addressed before drawing conclusions as have been provided. Contrary to the title of the article, not the entire spectrum of neurological disease is included in the discussion but only a few aspects. It is still unknown if hyposmia or myalgia are the most frequent neurological manifestations of the infection. Since only few epidemiological data are available about neurological disease in COVID-19 patients, it is currently too early to conclude that neurological compromise is rare. Whether the virus crosses the blood-brain barrier or not is also a matter of debate.

\section{Authors' Contributions}

All authors contributed equally to this study.

\section{Conflict of Interest Disclosures}

The authors declare no conflicts of interest

\section{Ethical Approval}

Informed consent was obtained from patients. Also the study was approved by the institutional review board.

\section{References}

1. Assari S. COVID-19 Pandemic and Neurological Disease: A Critical Review of the Existing Literature. Hosp Pract Res. 2020;5(3):81-86. doi:10.34172/hpr.2020.16

2. Utukuri PS, Bautista A, Lignelli A, Moonis G. Possible acute disseminated encephalomyelitis related to severe acute respiratory syndrome coronavirus 2 infection. AJNR Am J Neuroradiol. 2020; 41:E82-E83. doi:10.3174/ajnr.A6714.

3. Finsterer J, Stollberger C. Update on the neurology of COVID-19. J Med Virol 2020;92(11): 2316-2318. doi: 10.1002/jmv.26000.

4. Finsterer J, Scorza FA. SARS-CoV-2 myopathy. J Med Virol 2020; In Press. doi:10.1002/jmv.26550. 